\title{
Solid-state Photochemistry of Nitro Compounds: Structure-Reactivity Correlations
}

\author{
Kaillathe Padmanabhan, Dietrich Döpp, ${ }^{b}$ Kailasam Venkatesan," and Vaidyanathan Ramamurthy" \\ a Department of Organic Chemistry, Indian Institute of Science, Bangalore-560 012, India \\ b F.B.6. Organische Chemie, Universität Duisburg. D-4100, Duisburg-1, West Germany
}

The molecular structures of 1 - $\mathrm{t}$-butyl-3,5-dimethyl-2,4,6-trinitrobenzene, 1 - $\mathrm{t}$-butyl-3,4,5-trimethyl-2,6dinitrobenzene, and 1-t-butyl-4-acetyl-3,5-dimethyl-2,6-dinitrobenzene have been determined by single-crystal $X$-ray analyses with a view to establishing a structure-reactivity relationship in the photochemical intramolecular hydrogen-abstraction process in the solid state. The reactivity of these aromatic nitro compounds in the solid state has been rationalized in terms of relevant intramolecular geometrical parameters as well as intermolecular packing considerations.

Certain 2-nitro-t-butylbenzenes (1) (Scheme) upon $n \pi *$ excitation by u.v. light ( $\geqslant 280 \mathrm{~nm}$ ) undergo an intramolecular hydrogen abstraction. ${ }^{1-3}$ This primary reaction initiates a sequence of events $(2) \longrightarrow(3) \longrightarrow$ (4) leading to $3 \mathrm{H}$-indole 1 oxides as the final products (Scheme). Owing to both their thermal and photochemical instability, the $N$-oxides (4) have, however, not been isolated in most cases. Instead, the hydroxamic acids (5), derived from the hydrates of (4) by dehydrogenation, are generally obtained along with products of deoxygenation or isomerization of (4). Although this kind of photocyclization is not shown by most electron-donorsubstituted 2-nitro-t-butylbenzenes which lack lowlying $n, \pi^{*}$ excited states, compounds (6)-(8) (Scheme) show photobehaviour similar to that of (1). Furthermore, solid representatives of (1) $)^{2.5}$ as well as compounds (6)-(8) have been successfully converted into hydroxamic acids (5) by irradiation of their crystals followed by alkaline oxidative workup. ${ }^{4}$ It is noteworthy that compounds (6)-(8) do not undergo intramolecular hydrogen abstraction from the benzylic methyl groups, a normal photoreaction of ortho-nitrotoluenes, ${ }^{6}$ to any measurable extent. Instead, intramolecular abstraction from the non-activated $\beta$-position of the t-butyl group is clearly preferred. In this connection, we have carried out $X$-ray crystallographic studies on (6)-(8) aimed at gathering information about the photochemical hydrogen-abstraction process of aromatic nitro compounds. The correlation of solidstate chemical reactivity with $X$-ray crystal structure data has provided valuable insight into a variety of organic reaction types. In this connection, it may be noted that the correlation of $X$-ray structure with reactivity in the photochemical intramolecular hydrogen abstraction of ketones has been recently examined by Scheffer et al. and Trotter. ${ }^{7}$ Conclusions drawn from the present study are also expected to be of general interest. Results pertaining to structure and solid-state reactivity of (6)-(8) are discussed.

\section{Results and Discussion}

One would anticipate that the ideal geometry for the hydrogen abstraction by the $n \pi^{*}$ excited nitro group would be the following. In general, for a hydrogen atom to be abstracted by the oxygen atom of the nitro group, the favourable condition is an $\mathrm{O} \cdot . \mathrm{H}$ distance less than the sum of the van der Waals radii of these two atoms with the $\mathrm{C}-\mathrm{H}$ bond in the plane of the nitro group. Since the abstraction involves the $n \pi *$ excited state of the nitro group, in which the new $\mathrm{O}-\mathrm{H}$ bond is formed using the half occupied oxygen $n$-orbital, the ideal values for the intramolecular angles $\mathrm{C}-\mathrm{H} \ldots \mathrm{O}$ and $\mathrm{N}-\mathrm{O} \ldots \mathrm{H}$ should be $180^{\circ}$ and $90^{\circ}$, respectively. The next step, which involves the

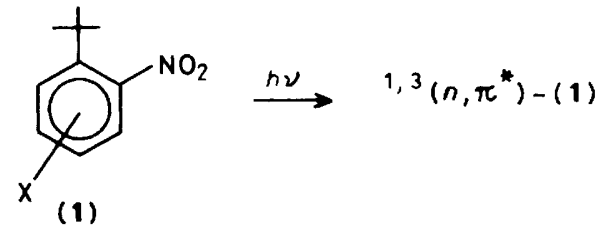

$$
\begin{aligned}
& X=\mathrm{H}, 4-\mathrm{But} \\
& 4-\text { and } 5-\mathrm{Ph} \\
& 4-\mathrm{Br}, 4-\mathrm{OMe}, \\
& 4-\mathrm{NHAC}, \\
& 4-\mathrm{CO}_{2} \mathrm{H}, 4-\mathrm{CN}, \\
& \text { and } 4-\mathrm{NO}_{2}
\end{aligned}
$$<smiles>[X]c1ccc([N+](=O)[O-])c(C(C)(C)C)c1</smiles>

(3)<smiles>[14CH3][14CH3]</smiles><smiles>[X]c1ccc2c(c1)C(C)(C)C[N+]2[O-]</smiles>

(4)<smiles>Cc1c(C)c([N+](=O)[O-])c(C(C)(C)C)c([N+](=O)[O-])c1C</smiles>

(2)<smiles>[X]c1ccc2c(c1)C(C)(C)C(=O)N2O</smiles>

(5)

$$
\begin{aligned}
& \text { (6) } Y=\mathrm{NO}_{2} \\
& \text { (7) } Y=M e \\
& \text { (8) } Y=A C
\end{aligned}
$$

Scheme. combination of the resulting $\mathrm{C}$ and $\mathrm{N}$ radical centres in (2) to yield (3) (Scheme) will again be favoured by a $\mathrm{C} \ldots \mathrm{N}$ intramolecular contact less than the sum of the van der Waals radii of $C$ and $N$ atoms. Structural details of $(6)-(8)$, based on 


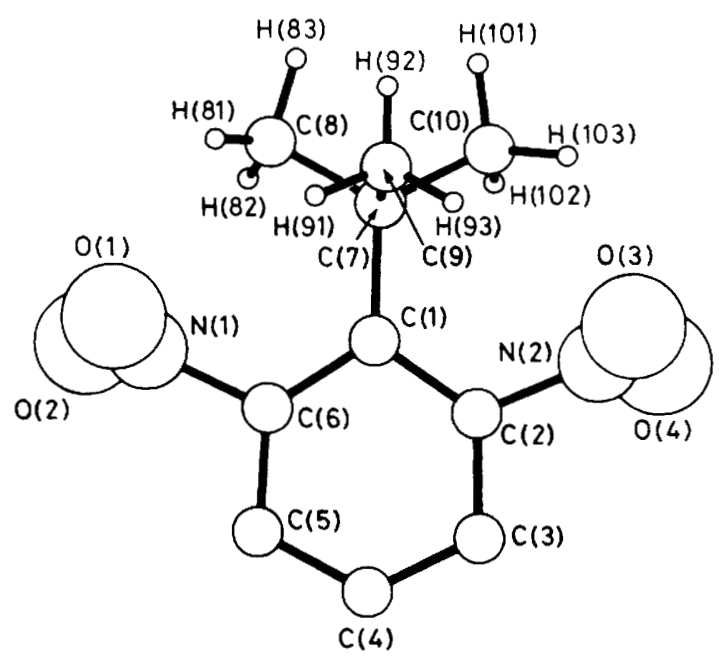

Figure 1. General numbering scheme for the reactive groups for compounds (6) and (7) and molecules A, B and D of compound (8)

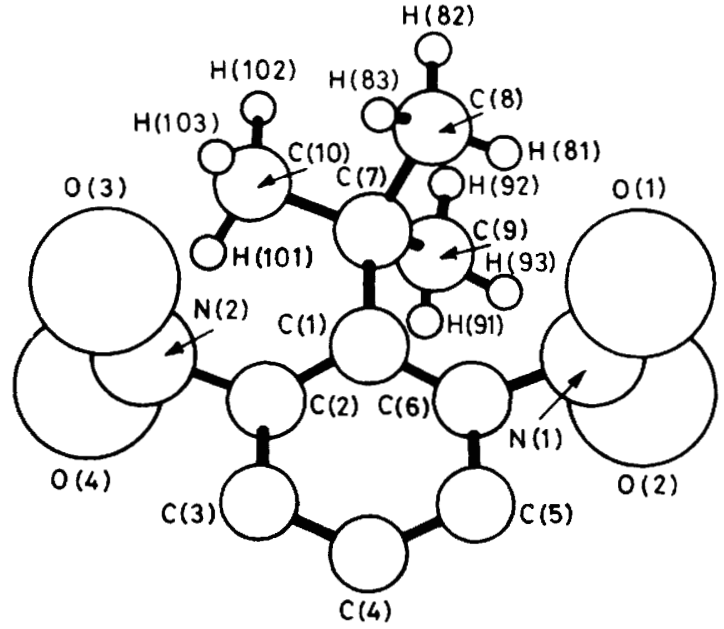

Figure 2. Numbering scheme for the reactive groups for molecule $\mathrm{C}$ of compound (8)

Table 1. Intramolecular $\mathrm{O} \ldots \mathrm{H}$ contacts $(\AA)(\leqslant 3 \AA)$

\begin{tabular}{|c|c|c|c|c|c|}
\hline \multirow[b]{2}{*}{$\mathbf{O} \cdots \mathrm{H}^{a}$} & \multirow[b]{2}{*}{ Compd. (6) } & \multirow[b]{2}{*}{ Compd. (7) ${ }^{b}$} & \multicolumn{3}{|c|}{ Compd. $(8)^{b}$} \\
\hline & & & Molecule A & Molecule B & Molecule D \\
\hline$O(1) \cdots H(91)$ & 2.57 & 2.51 & 2.60 & 2.55 & 2.74 \\
\hline$O(3) \cdots H(93)$ & 2.74 & 2.67 & 2.56 & 2.58 & 2.51 \\
\hline $\mathrm{O}(1) \cdots \mathrm{H}(81)$ & 2.41 & 2.31 & 2.31 & 2.27 & 2.37 \\
\hline $\mathrm{O}(2) \cdots H(82)$ & 2.90 & 2.66 & 2.40 & 3.00 & 2.72 \\
\hline $\mathrm{O}(2) \cdots H(81)$ & 2.45 & 2.46 & 2.58 & 2.37 & 2.45 \\
\hline$O(3) \ldots H(103)$ & 2.42 & 2.30 & 2.49 & 2.22 & 2.29 \\
\hline $\mathrm{O}(4) \cdots \mathrm{H}(102)$ & 2.57 & 2.89 & 2.96 & 2.90 & 2.64 \\
\hline$O(4) \cdots H(103)$ & 2.68 & 2.49 & 2.68 & 2.35 & 2.42 \\
\hline
\end{tabular}

" The van der Waals sum for $\mathrm{O} \ldots \mathrm{H}=2.6 \AA{ }^{b}$ The e.s.d. for (6) and (7) is about $0.03 \AA$ and for (8) it is $0.21 \AA$.

Table 2. Intramolecular $\mathrm{C} \cdots \mathrm{N}$ contacts $(\AA)$

\begin{tabular}{|c|c|c|c|c|c|}
\hline \multirow[b]{2}{*}{$C \cdots N^{a}$} & \multirow[b]{2}{*}{ Compd. (6) } & \multirow[b]{2}{*}{ Compd. $(7)^{b}$} & \multicolumn{3}{|c|}{ Compd. $(8)^{b}$} \\
\hline & & & Molecule A & Molecule B & Molecule D \\
\hline$C(9) \cdots N(1)$ & 3.550 & 3.576 & 3.654 & 3.641 & 3.599 \\
\hline$C(9) \ldots N(2)$ & 3.640 & 3.642 & 3.532 & 3.549 & 3.595 \\
\hline$C(8) \cdots N(1)$ & 2.781 & 2.786 & 2.830 & 2.771 & 2.791 \\
\hline$C(10) \cdots N(2)$ & 2.773 & 2.783 & 2.739 & 2.817 & 2.815 \\
\hline
\end{tabular}

a The van der Waals sum for $C \cdots N=3.3 \AA$. ${ }^{b}$ The e.s.d. for (6) and (7) is about $0.005 \AA$ and for (8) it is $0.024 \AA$.

our $X$-ray crystallographic studies and relevant for the photochemical process under consideration, are summarized below.

The general numbering scheme for the groups involved in the reaction (the t-butyl group and the two ortho-nitro groups) is shown in Figure 1 for (6), (7), and molecules A, B, and D of (8) [the unit cell of (8) contains four independent molecules in the asymmetric unit] and in Figure 2 for molecule $C$ of (8). Intramolecular geometrical parameters obtained from $X$-ray crystallographic studies involving (i) hydrogens of the t-butyl group and oxygens of the two adjacent nitro groups ( $\leqslant 3 \AA$ ), (ii) methyl carbon atoms of the t-butyl group and nitrogen atoms of the nitro group, (iii) the intramolecular $\mathrm{C}-\mathrm{H} \ldots \mathrm{O}$ angles, and (iv) the intramolecular $\mathrm{N}-\mathrm{O} \ldots \mathrm{H}$ angles, are provided in Tables 1, 2, 3, and 4 respectively, for (6), (7), and molecules A, B, and D of (8), and in Table 6 for molecule $C$ of (8).

For the sake of brevity, only the results of (6) are discussed at length and those of (7) and (8) are appropriately highlighted. Nitro groups in (6) - (8) in principle can be expected to abstract a hydrogen either from the $t$-butyl group or from the benzylic methyl group. Based on the assumption that short intramolecular O $\ldots H$ contacts would favour hydrogen abstraction, eight possibilities for hydrogen-abstraction from the t-butyl group by two adjacent nitro groups exist in the case of (6) (Table 1). But when we consider the geometrical requirement for such a hydrogen-abstraction reaction, i.e. the $\mathrm{C}-\mathrm{H} \cdot \cdots \mathrm{O}$ and $\mathrm{N}-\mathrm{O} \cdots \mathrm{H}$ angles (Tables 3 and 4 respectively), the eight possibilities are reduced to two: $H(91)$ could be abstracted by $O(1)$ and $H(93)$ by $O(3)$. This reduction in number, based on structural parameters, is remarkable considering the fact that there are 36 possible modes of hydrogen abstraction. Additional evidence for the choice of the above two sets of atoms comes from the following consideration. Hypothetical points were fixed in the direction of the 
Table 3. Intramolecular $\mathrm{C}-\mathrm{H} \cdot \ldots \mathrm{O}$ angles ( $\left(^{\circ}\right)$

\begin{tabular}{|c|c|c|c|c|c|}
\hline \multirow{2}{*}{$\mathrm{C}-\mathrm{H} \cdots \mathrm{O}^{a}$} & \multirow[b]{2}{*}{ Compd. (6) ${ }^{b}$} & \multirow{2}{*}{ Compd. $(7)^{b}$} & \\
\hline & & & Molecule A & Molecule B & Molecule D \\
\hline$C(9)-H(91) \cdots O(1)$ & 135.8 & 140.4 & 130.9 & 141.0 & 124.9 \\
\hline $\mathrm{C}(9)-\mathrm{H}(93) \ldots \mathrm{O}(3)$ & 129.5 & 134.3 & 137.8 & 130.9 & 144.3 \\
\hline $\mathrm{C}(8)-\mathrm{H}(81) \ldots \mathrm{O}(1)$ & 123.6 & 137.1 & 134.2 & 137.0 & 130.8 \\
\hline$C(8)-H(82) \ldots O(2)$ & 87.1 & 97.4 & 108.8 & 80.8 & 103.0 \\
\hline $\mathrm{C}(8)-\mathrm{H}(81) \ldots \mathrm{O}(2)$ & 112.7 & 106.1 & 102.6 & 113.3 & 110.8 \\
\hline$C(10)-H(103) \ldots O(3)$ & 130.4 & 129.2 & 124.3 & 139.2 & 140.6 \\
\hline$C(10)-H(102) \ldots O(4)$ & 105.9 & 85.1 & 84.6 & 83.1 & 100.0 \\
\hline$C(10)-H(103) \ldots O(4)$ & 100.1 & 103.1 & 95.7 & 110.9 & 112.3 \\
\hline
\end{tabular}

${ }^{a}$ The ideal value of $\mathrm{C}-\mathrm{H} \cdots \mathrm{O}=180^{\circ} .{ }^{b}$ The e.s.d. for $(6)=2.2^{\circ},(7)=0.9^{\circ}$, and for $(8)=13.1^{\circ}$.

Table 4. Intramolecular $\mathrm{N}-\mathrm{O} \cdots \mathrm{H}$ angles $\left({ }^{\circ}\right)$

\begin{tabular}{|c|c|c|c|c|c|}
\hline \multirow[b]{2}{*}{$\mathrm{N}-\mathrm{O} \cdots \mathrm{H}^{a}$} & \multirow[b]{2}{*}{ Compd. $(6)^{b}$} & \multirow[b]{2}{*}{ Compd. $(7)^{b}$} & \multicolumn{3}{|c|}{ Compd. (8) ${ }^{b}$} \\
\hline & & & Molecule A & Molecule B & Molecule D \\
\hline $\mathrm{N}(1)-\mathrm{O}(1) \cdots \mathrm{H}(91)$ & 100.4 & 99.2 & 100.7 & 100.2 & 102.7 \\
\hline $\mathrm{N}(2)-\mathrm{O}(3) \cdots \mathrm{H}(93)$ & 101.4 & 100.0 & 100.0 & 102.2 & 99.9 \\
\hline $\mathrm{N}(1)-\mathrm{O}(1) \cdots \mathrm{H}(81)$ & 67.5 & 67.5 & 70.9 & 66.8 & 67.1 \\
\hline $\mathrm{N}(1)-\mathrm{O}(2) \cdots \mathrm{H}(82)$ & 82.9 & 83.4 & 86.3 & 85.5 & 79.8 \\
\hline $\mathrm{N}(1)-\mathrm{O}(2) \cdots \mathrm{H}(81)$ & 65.9 & 61.0 & 59.8 & 62.5 & 64.0 \\
\hline $\mathrm{N}(2)-\mathrm{O}(3) \cdots \mathrm{H}(103)$ & 71.5 & 70.4 & 71.0 & 66.2 & 66.2 \\
\hline $\mathrm{N}(2)-\mathrm{O}(4) \cdots \mathrm{H}(102)$ & 83.1 & 86.0 & 81.8 & 82.5 & 84.0 \\
\hline $\mathrm{N}(2) \mathrm{O}(4) \cdots \mathrm{H}(103)$ & 60.7 & 62.8 & 63.3 & 61.0 & 61.1 \\
\hline
\end{tabular}

${ }^{a}$ The ideal value of $\mathrm{N}-\mathrm{O} \ldots \mathrm{H}=90^{\circ} .{ }^{b}$ The e.s.d. for $(6)=0.7^{\circ},(7)=0.3^{\circ}$, and for $(8)=4.8^{\circ}$.

Table 5. O $\ldots$ HYP $\ldots$ H Angles $\left({ }^{\circ}\right)$

$\begin{array}{cccccc}\text { O } \cdots \text { HYP } \ldots \text { H }^{a} & \text { Compd. (6) } & \text { Compd. (7) } & \overbrace{\text { Molecule A }} & \text { Molecule B } & \text { Molecule D } \\ \text { O(1) } \cdots \text { HYP } \ldots \text { H(91) } & 111.8(111.7) & 100.5(100.9) & 111.6(111.8) & 107.0(107.4) & 105.8(108.3) \\ \text { O(3) } \cdots \text { HYP } \ldots \text { H(93) } & 107.8(109.2) & 103.0(103.8) & 104.0(104.6) & 101.9(104.3) & 103.2(103.8) \\ \text { O(1) } \cdots \text { HYP } \cdots \text { H(81) } & 55.8(48.2) & 57.7(49.9) & 59.3(52.7) & 58.0(49.9) & 57.1(49.2) \\ \text { O(2) } \cdots \text { HYP } \ldots \text { H(82) } & 87.7(81.8) & 88.9(82.7) & 79.1(75.7) & 90.9(85.8) & 84.6(77.7) \\ \text { O(2) } \cdots \text { HYP } \ldots \text { H(81) } & 55.4(47.3) & 56.3(46.2) & 56.7(46.0) & 57.0(47.4) & 56.4(47.4) \\ \text { O(3) } \cdots \text { HYP } \ldots \text { H(103) } & 61.2(54.5) & 55.8(49.4) & 59.2(52.6) & 56.4(48.3) & 59.4(51.0) \\ \text { O(4) } \cdots \text { HYP } \cdots \text { H(102) } & 82.6(77.4) & 87.3(82.9) & 90.6(83.7) & 93.9(86.6) & 89.6(83.6) \\ \text { O(4) } \cdots \text { HYP } \cdots \text { H(103) } & 58.5(47.8) & 54.2(44.9) & 57.5(48.0) & 55.4(45.4) & 58.0(47.8)\end{array}$

${ }^{a}$ Ideal value of $\mathrm{O} \cdots \mathrm{HYP} \cdots \mathrm{H}=180^{\circ}$. The values outside the parentheses correspond to $\mathrm{N}-\mathrm{O} \cdots \mathrm{HYP}=90^{\circ}$ and those inside to $\mathrm{N}-\mathrm{O} \cdots \mathrm{HYP}=$ $120^{\circ}$.

Table 6. Intramolecular geometric parameters for molecule $\mathrm{C}$ of (8)

$$
\begin{array}{lr}
O \cdots H \text { Contacts }(\AA)^{a, b} \\
\text { O(1) } \cdots H(81) & 2.22 \\
O(2) \cdots H(93) & 2.24 \\
O(3) \cdots H(101) & 2.93 \\
O(3) \cdots H(103) & 2.28 \\
O(4) \cdots H(101) & 2.64
\end{array}
$$

$$
\mathrm{C}-\mathrm{H} \cdots \mathrm{O} \text { Angles }\left({ }^{\circ}\right)^{d}
$$

$\mathrm{C}(8)-\mathrm{H}(81) \ldots \mathrm{O}(1)$

C(9)-H(93) $\ldots O$ O(2) $\quad 153.3$

$\mathrm{C}(10)-\mathrm{H}(101) \cdots \mathrm{O}(3) \quad 86.4$

$\mathrm{C}(10)-\mathrm{H}(103) \cdots O(3) \quad 122.9$
Compd. (8)

a For numbering scheme, see Figure $2{ }^{b}$ The van der Waals sum for $\mathrm{O} \cdots \mathrm{H}=2.6 \AA .{ }^{c}$ The van der Waals sum for $\mathrm{C} \cdots \mathrm{N}=3.3 \AA .{ }^{d}$ Ideal value of $\mathrm{C}-\mathrm{H} \cdots \mathrm{O}=180^{\circ}$. ${ }^{e}$ Ideal value of $\mathrm{N}-\mathrm{O} \cdots \mathrm{H}=90^{\circ}$.

lone-pair orbitals of the nitro group at $90^{\circ}$ at a distance of $1 \AA$ from the oxygen atoms $O(1), O(2), O(3)$, and $O(4)$. For hydrogen abstraction, the favourable condition would be collinearity of the atoms $O$, hypothetical point (represented by HYP) and $H$. The angles $O \ldots$ HYP .... H for the eight possibilities are recorded in Table 5. Even though these values deviate from collinearity for the above two choices, the deviations are more for the other six sets. Similar calculations under the supposition that the non-bonding orbitals may be orientated at $120^{\circ}$ instead of $90^{\circ}$ were also carried out and the results are substantially the same as those for the $90^{\circ}$ calculation.

We attempted to explore whether it would be possible to make a unique identification of the hydrogen being abstracted. The intramolecular geometrical criteria do not seem to provide any information regarding this, the reason being that the methyl group under consideration, i.e. 9-Me, is nearly symmetrical with 
Table 7. Sum of $\left(d_{0}-d_{c}\right)^{2}$ for rotation of the t-butyl group-compounds (6) and (7)

\begin{tabular}{|c|c|c|c|c|c|c|c|}
\hline \multicolumn{4}{|c|}{ Compd. (6) } & \multicolumn{4}{|c|}{ Compd. (7) } \\
\hline \multicolumn{2}{|c|}{ Rotation + ve direction } & \multicolumn{2}{|c|}{ Rotation - ve direction } & \multicolumn{2}{|c|}{ Rotation + ve direction } & \multicolumn{2}{|c|}{ Rotation - ve direction } \\
\hline Interval $\left({ }^{\circ}\right)$ & $S=\Sigma\left(d_{0}-d_{c}\right)^{2}$ & Interval $\left({ }^{\circ}\right)$ & $S=\Sigma\left(d_{0}-d_{c}\right)^{2}$ & Interval $\left({ }^{\circ}\right)$ & $S=\Sigma\left(d_{0}-d_{c}\right)^{2}$ & Interval $\left({ }^{\circ}\right)$ & $S=\Sigma\left(d_{0}-d_{c}\right)^{2}$ \\
\hline 5 & 0 & -5 & 0 & 5 & 0.0081 & -5 & 0 \\
\hline 10 & 0 & -10 & 0.0001 & 10 & 0.0309 & -10 & 0 \\
\hline 15 & 0 & -15 & 0.0169 & 15 & 0.0622 & -15 & 0.0016 \\
\hline 20 & 0 & -20 & 0.0562 & 20 & 0.0835 & -20 & 0.0102 \\
\hline 25 & 0 & -25 & 0.1356 & 25 & 0.0978 & -25 & 0.0451 \\
\hline 30 & 0.0016 & -30 & 0.2440 & 30 & 0.0982 & -30 & 0.0916 \\
\hline
\end{tabular}

Table 8. Torsion angles $\left({ }^{\circ}\right)$

\begin{tabular}{|c|c|c|c|c|c|c|}
\hline \multirow[b]{2}{*}{ Bond } & \multirow[b]{2}{*}{ Compd. $(6)^{a}$} & \multirow[b]{2}{*}{ Compd. (7) ${ }^{a}$} & \multicolumn{4}{|c|}{ Compd. (8) } \\
\hline & & & Molecule $\mathrm{A}^{a}$ & Molecule Ba & Molecule $\mathrm{C}^{b}$ & Molecule $\mathrm{D}^{a}$ \\
\hline$C(2)-C(1)-C(7)-C(8)$ & -148.7 & 148.0 & -155.9 & -150.2 & 116.3 & 152.4 \\
\hline$C(2)-C(1)-C(7)-C(9)$ & 91.3 & -90.1 & 85.9 & 87.0 & -120.9 & -88.2 \\
\hline$C(2)-C(1)-C(7)-C(10)$ & -30.5 & 30.5 & -37.5 & -32.2 & -3.7 & 35.3 \\
\hline $\mathrm{C}(1)-\mathrm{C}(2)-\mathrm{N}(2)-\mathrm{O}(3)$ & -79.3 & 81.2 & -80.9 & -79.3 & 93.7 & 78.8 \\
\hline $\mathrm{C}(1)-\mathrm{C}(2)-\mathrm{N}(2)-\mathrm{O}(4)$ & 103.5 & -101.6 & 102.5 & 103.9 & -90.4 & -102.8 \\
\hline $\mathrm{C}(1)-\mathrm{C}(6)-\mathrm{N}(1)-\mathrm{O}(1)$ & 77.6 & -81.4 & 81.0 & 78.3 & 91.5 & -77.0 \\
\hline $\mathrm{C}(1)-\mathrm{C}(6)-\mathrm{N}(1)-\mathrm{O}(2)$ & -105.3 & 102.8 & -102.6 & -102.4 & -91.1 & 105.5 \\
\hline
\end{tabular}

${ }^{a}$ Numbering scheme in Figure 1. ${ }^{b}$ Numbering scheme in Figure 2.

Table 9. Crystal data for compounds (6), (7), and (8)

\begin{tabular}{|c|c|c|c|}
\hline & Compd. (6) & Compd. (7) & Compd. (8) \\
\hline Molecular formula & $\mathrm{C}_{12} \mathrm{H}_{15} \mathrm{~N}_{3} \mathrm{O}_{6}$ & $\mathrm{C}_{13} \mathrm{H}_{18} \mathrm{~N}_{2} \mathrm{O}_{4}$ & $\mathrm{C}_{14} \mathrm{H}_{18} \mathrm{~N}_{2} \mathrm{O}_{5}$ \\
\hline$M_{r}$ & 297.3 & 266.3 & 294.3 \\
\hline Space group & Monoclinic, $P 2_{1} / a$ & Monoclinic, $P 2_{1} / n$ & Monoclinic, $P 2_{1} / n$ \\
\hline$a(A)$ & $9.220(1)$ & $9.610(1)$ & $24.369(4)$ \\
\hline$b(\mathrm{~A})$ & $11.104(1)$ & $14.619(1)$ & $7.834(1)$ \\
\hline$c(\AA)$ & $14.622(2)$ & $11.006(1)$ & $31.956(5)$ \\
\hline$\alpha\left({ }^{\circ}\right)$ & 90 & 90 & 90 \\
\hline$\beta\left({ }^{\circ}\right)$ & $107.79(1)$ & $115.43(1)$ & $97.39(1)$ \\
\hline$\gamma\left({ }^{\circ}\right)$ & 90 & 90 & 90 \\
\hline$V\left(\AA^{3}\right)$ & $1426.3(3)$ & $1396.3(1)$ & $6050.2(15)$ \\
\hline$Z$ & 4 & 4 & 16 \\
\hline $\bar{D}_{\mathrm{m}}\left(\mathrm{Mg} \mathrm{m}^{-3}\right)$ & 1.365 & 1.243 & 1.280 \\
\hline$D_{\mathrm{x}}\left(\mathrm{Mg} \mathrm{m}^{-3}\right)$ & 1.384 & 1.267 & 1.292 \\
\hline Radiation used & Mo- $K_{\alpha}$ & $\mathrm{Cu}-K_{\alpha}$ & $\mathrm{Mo}-K_{\alpha}$ \\
\hline$\lambda(\AA)$ & 0.7107 & 1.5418 & 0.7107 \\
\hline$\mu\left(\mathrm{cm}^{-1}\right)$ & 0.72 & 6.99 & 0.61 \\
\hline$F(000)$ & 624.0 & 568.0 & 2496.0 \\
\hline Crystal size (mm) & $0.31 \times 0.09 \times 0.09$ & $0.12 \times 0.25 \times 0.24$ & $0.25 \times 0.12 \times 0.10$ \\
\hline$\theta$ limit $\left({ }^{\circ}\right)$ & 30 & 75 & 23 \\
\hline Mode of data collection & $\omega / 2 \theta$ & $\omega / 2 \theta$ & $\omega / 2 \theta$ \\
\hline No. of intensity controls & 3 & 3 & 3 \\
\hline Total no. of reflections collected & 4577 & 2867 & 8384 \\
\hline No. of observed reflections & 1591 & 1869 & 4170 \\
\hline & $\left(\left|F_{\mathrm{o}}\right| \geqslant 4.5 \sigma\left|F_{\mathrm{o}}\right|\right)$ & $\left(\left|F_{\mathrm{o}}\right| \geqslant 6 \sigma\left|F_{\mathrm{o}}\right|\right)$ & $\left(\left|F_{\mathrm{o}}\right| \geqslant 3 \sigma\left|F_{\mathrm{o}}\right|\right)$ \\
\hline Final $R$ & 0.0497 & 0.0592 & 0.073 \\
\hline Weighted $R\left(R_{w}\right)$ & 0.0709 & 0.0776 & 0.115 \\
\hline $\begin{array}{l}\text { Weighting function (w) } \\
\text { No of variables }\end{array}$ & $\begin{array}{c}1.5965 /\left(\sigma^{2}|F|+0.002|F|^{2}\right) \\
250\end{array}$ & $\begin{array}{c}4.0125 /\left(\sigma^{2}|F|+0.000375|F|^{2}\right) \\
244\end{array}$ & 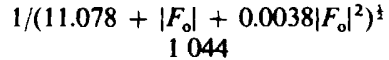 \\
\hline
\end{tabular}

respect to the two ortho nitro groups. Therefore, it was felt that the consideration of the next step, namely the combination of the radical centres in (2) (Scheme), may provide a useful clue. For an ideal reaction one would expect that the distance between the radical centres in (2) would be short. In terms of the ground-state structure of (6), in order to obtain the observed product it would be desirable to have a distance less than the sum of the van der Waals radii for $C(9)$... N(1) and $C(9)$... $\mathrm{N}(2)$. However, these distances are quite large as shown in Table 2. It should be kept in mind that the geometry and structure of the diradical (2) would be expected to differ from that of the ground-state molecules and the conclusions drawn based on the latter may be taken as only a rough guide in explaining the solid-state reactivity. 
Table 10. F ractional atomic co-ordinates $\left(\times 10^{4}\right)$ for non-hydrogen atoms in compounds (6)-(8) with their e.s.d.s in parentheses

Compound (6)

$\begin{array}{lcrc}\text { Atom } & x & y & z \\ \mathrm{C}(1) & 8129(3) & 425(2) & 7284(2) \\ \mathrm{C}(2) & 7893(3) & 561(2) & 8182(2) \\ \mathrm{C}(3) & 7835(3) & 1642(2) & 8659(2) \\ \mathrm{C}(4) & 8010(3) & 2672(2) & 8161(2) \\ \mathrm{C}(5) & 8265(3) & 2677(2) & 7285(2) \\ \mathrm{C}(6) & 8307(3) & 1540(2) & 6877(2) \\ \mathrm{C}(7) & 8129(3) & -808(2) & 6778(2) \\ \mathrm{C}(8) & 6502(4) & -1045(4) & 6138(3) \\ \mathrm{C}(9) & 9204(4) & -826(3) & 6151(3) \\ \mathrm{C}(10) & 8681(6) & -1860(3) & 7480(3) \\ \mathrm{C}(11) & 8471(4) & 3831(3) & 6782(3) \\ \mathrm{C}(12) & 7586(5) & 1698(3) & 9626(2) \\ \mathrm{N}(13) & 8504(3) & 1613(2) & 5914(2) \\ \mathrm{N}(14) & 7586(3) & -493(2) & 8713(2) \\ \mathrm{N}(15) & 7881(3) & 3841(2) & 8604(2) \\ 1 O(13) & 7363(3) & 1495(2) & 5217(1) \\ 2 O(13) & 9770(3) & 1834(2) & 5859(2) \\ 1 O(14) & 6295(3) & -894(2) & 8459(3) \\ 2 O(14) & 8626(3) & -861(2) & 9391(2) \\ 1 O(15) & 6661(3) & 4327(2) & 8367(2) \\ 2 O(15) & 8978(3) & 4248(2) & 9198(2)\end{array}$

Compound (7)

$\begin{array}{lr}C(1) & 11205(2) \\ C(2) & 10886(2) \\ C(3) & 9589(2) \\ C(4) & 8500(2) \\ C(5) & 8747(2) \\ C(6) & 10059(2) \\ C(7) & 12661(2) \\ C(8) & 12335(4) \\ C(9) & 13289(3) \\ C(10) & 13926(3) \\ C(11) & 7602(3) \\ C(12) & 7041(3) \\ C(13) & 9370(3) \\ N(14) & 10188(2) \\ N(15) & 11980(2) \\ 1 O(14) & 10998(3) \\ 2 O(14) & 9411(3) \\ 1 O(15) & 11685(2) \\ 2 O(15) & 13087(2) \\ C\end{array}$

Compound (8), Molecule A

$\begin{array}{llll}\mathrm{C}(1) & 7319(5) & 4100(17) & 3249(4) \\ \mathrm{C}(2) & 7147(5) & 5572(17) & 3454(4) \\ \mathrm{C}(3) & 6661(5) & 6479(17) & 3354(4) \\ \mathrm{C}(4) & 6292(6) & 5877(17) & 3018(4) \\ \mathrm{C}(5) & 6412(6) & 4424(19) & 2795(4) \\ \mathrm{C}(6) & 6912(6) & 3641(18) & 2915(5) \\ \mathrm{C}(7) & 7860(6) & 3129(19) & 3387(5) \\ \mathrm{C}(8) & 7742(8) & 1838(27) & 3729(7) \\ \mathrm{C}(9) & 8350(7) & 4311(26) & 3548(8) \\ \mathrm{C}(10) & 8065(8) & 2107(26) & 3025(7) \\ \mathrm{C}(11) & 6000(7) & 3734(22) & 2436(5) \\ \mathrm{C}(12) & 6497(7) & 7981(20) & 3603(5) \\ \mathrm{C}(13) & 5739(6) & 6757(21) & 2909(5) \\ \mathrm{C}(14) & 5692(7) & 8092(23) & 2578(6) \\ \mathrm{N}(1) & 6982(6) & 2062(18) & 2668(4) \\ \mathrm{N}(2) & 7491(5) & 6249(18) & 3833(4) \\ \mathrm{O}(1) & 6859(6) & 714(14) & 2819(5) \\ O(2) & 7130(7) & 2222(19) & 2323(4) \\ O(3) & 7448(6) & 5550(20) & 4165(4) \\ O(4) & 7774(5) & 7508(15) & 3788(5) \\ O(5) & 5364(5) & 6387(19) & 3100(5)\end{array}$

Table $1^{n}$ (continued)

Compound (8), Molecule B

Atom

$\mathrm{C}(1)$

$C(2)$
$C(3)$

$\mathrm{C}(4)$

$\mathrm{C}(5)$

$\mathrm{C}(6)$

$\mathrm{C}(7)$

$\mathrm{C}(8)$

$\mathrm{C}(9)$

$\mathrm{C}(10)$

C(12)

$\mathrm{C}(13)$

$\mathrm{C}(14)$

$\mathrm{N}(1)$

$N(2)$

$O(1)$

$\mathrm{O}(2)$

$\mathrm{O}(3)$

$\mathrm{O}(4)$

$x$
$10258(5)$
$10435(6)$
$10919(6)$
$11281(6)$
$11149(6)$
$10649(6)$
$9702(6)$
$9792(8)$
$9236(7)$
$9464(9)$
$11566(7)$
$11072(8)$
$11821(6)$
$11854(8)$
$10582(7)$
$10102(6)$
$10442(7)$
$10683(7)$
$9828(6)$
$10141(5)$
$12202(5)$

$y$
$-868(17)$

$527(17)$

$1444(18)$

913(20)

$-521(20)$

$-1341(19)$

$-1823(20)$

$-3205(26)$

$-616(27)$

$-2609(31)$

$-1159(26)$

2 925(22)

$1848(22)$

3 089(29)

$-2867(20)$

$1150(18)$

$-2621(21)$

$-4235(16)$

$2453(17)$

$355(19)$

$1603(21)$

Compound (8), Molecule C

$\mathrm{C}(1)$

$12319(5)$

C(2)

$12486(5)$

$\mathrm{C}(3)$

C(4)

C(5)

C(6)

C(7)

$\mathrm{C}(8)$

C(9)

C(10)

C(12)

C(13)

C(14)

N(1)

$\mathrm{N}(2)$

$O(1)$

$\mathrm{O}(2)$

$\mathrm{O}(3)$

$\mathrm{O}(4)$
$\mathrm{O}(5)$

12 171(5)

$11643(6)$

$11441(6)$

11 792(6)

$12676(7)$

$12804(12)$

$12404(13)$

$13229(13)$

$10872(7)$

$12394(7)$

$11295(6)$

$10939(8)$

$11525(6)$

$13048(5)$

$11593(6)$

$11242(6)$

$13122(5)$

$13389(5)$

$11309(6)$

$-4301(16)$

$-5710(17)$

$-6559(17)$

$-5967(20)$

$-4546(20)$

$-3765(18)$

$-3393(19)$

$-1607(29)$

$-3437(73)$

$-4177(39)$

$-3850(27)$

$-8099(26)$

$-6795(23)$

$-8279(26)$

$-2260(17)$

$-6444(17)$

$-898(15)$

$-2517(19)$

$-7607(15)$

$-5853(24)$

$-6261(23)$

$3461(4)$

$3240(4)$

2 916(4)

$2787(4)$

2 966(5)

3 296(4)

$3829(5)$

$3729(8)$

4 204(7)

$3970(12)$

$2820(6)$

$2696(6)$

$2418(5)$

$2509(6)$

$3474(5)$

3 334(4)

$3317(5)$

$3754(5)$

$3577(4)$

3 133(5)

$2069(4)$

Compound (8), Molecule D

\begin{tabular}{llrl}
$\mathrm{C}(1)$ & $14186(5)$ & $848(17)$ & $5392(4)$ \\
$\mathrm{C}(2)$ & $14662(6)$ & $1350(18)$ & $5640(4)$ \\
$\mathrm{C}(3)$ & $14900(5)$ & $543(19)$ & $6007(5)$ \\
$\mathrm{C}(4)$ & $14636(5)$ & $-876(17)$ & $6143(4)$ \\
$\mathrm{C}(5)$ & $14151(5)$ & $-1481(17)$ & $5917(4)$ \\
$\mathrm{C}(6)$ & $13946(5)$ & $-612(17)$ & $5557(4)$ \\
$\mathrm{C}(7)$ & $13931(6)$ & $1829(19)$ & $4985(4)$ \\
$\mathrm{C}(8)$ & $13596(9)$ & $644(24)$ & $4663(5)$ \\
$\mathrm{C}(9)$ & $13543(8)$ & $3198(22)$ & $5116(6)$ \\
$\mathrm{C}(10)$ & $14370(9)$ & $2606(28)$ & $4737(6)$ \\
$\mathrm{C}(11)$ & $13854(6)$ & $-2978(21)$ & $6086(5)$ \\
$\mathrm{C}(12)$ & $15427(7)$ & $1236(26)$ & $6263(6)$ \\
$\mathrm{C}(13)$ & $14879(6)$ & $-1775(21)$ & $6546(4)$ \\
$\mathrm{C}(14)$ & $15322(8)$ & $-3075(28)$ & $6515(6)$ \\
$\mathrm{N}(1)$ & $13396(5)$ & $-1242(16)$ & $5369(4)$ \\
$\mathrm{N}(2)$ & $14978(6)$ & $2917(17)$ & $5545(4)$ \\
$\mathrm{O}(1)$ & $13384(6)$ & $-2537(14)$ & $5153(4)$ \\
$\mathrm{O}(2)$ & $12988(4)$ & $-462(18)$ & $5453(4)$ \\
$\mathrm{O}(3)$ & $14781(6)$ & $4276(15)$ & $5651(5)$ \\
$\mathrm{O}(4)$ & $15393(5)$ & $2741(17)$ & $5385(5)$ \\
$\mathrm{O}(5)$ & $14711(6)$ & $-1428(19)$ & $6872(3)$ \\
\hline
\end{tabular}


Table 11. Selected bond lengths involving non-hydrogen atoms in compounds (6) - (8) with their e.s.d.s in parentheses

\section{Compound (6)}

$\begin{array}{lc}\text { Atoms } & \text { Distance }(\AA) \\ \mathrm{C}(1)-\mathrm{C}(2) & 1.403(4) \\ \mathrm{C}(1)-\mathrm{C}(6) & 1.405(3) \\ \mathrm{C}(1)-\mathrm{C}(7) & 1.556(3) \\ \mathrm{C}(2)-\mathrm{C}(3) & 1.397(3) \\ \mathrm{C}(2)-\mathrm{N}(14) & 1.479(3) \\ \mathrm{C}(3)-\mathrm{C}(4) & 1.391(3) \\ \mathrm{C}(3)-\mathrm{C}(12) & 1.501(4) \\ \mathrm{C}(4)-\mathrm{C}(5) & 1.371(4) \\ \mathrm{C}(4)-\mathrm{N}(15) & 1.472(3) \\ \mathrm{C}(5)-\mathrm{C}(6) & 1.402(3) \\ \mathrm{C}(5)-\mathrm{C}(11) & 1.518(4)\end{array}$

Compound (7)

$\mathrm{C}(1)-\mathrm{C}(2)$

$\mathrm{C}(1)-\mathrm{C}(6)$

$\mathrm{C}(1)-\mathrm{C}(7)$

$\mathrm{C}(2)-\mathrm{C}(3)$

$\mathrm{C}(2)-\mathrm{N}(15)$

$\mathrm{C}(3)-\mathrm{C}(4)$

$C(3)-C(13)$

$\mathrm{C}(4)-\mathrm{C}(5)$

$\mathrm{C}(4)-\mathrm{C}(12)$

$\mathrm{C}(5)-\mathrm{C}(6)$

Compound (8)

Molecule A

$\mathrm{C}(1)-\mathrm{C}(2)$

$\mathrm{C}(1)-\mathrm{C}(6)$

C(1)-C(7)

$\mathrm{C}(2)-\mathrm{C}(3)$

$\mathrm{C}(2)-\mathrm{N}(2)$

C(3)-C(4)

C(3)-C(12)

C(4)-C(5)

C(4)-C(13)

$\mathrm{C}(5)-\mathrm{C}(6)$

C(5)-C(11)

$\mathrm{C}(6)-\mathrm{N}(1)$

$\mathrm{C}(7)-\mathrm{C}(8)$

$\mathrm{C}(7)-\mathrm{C}(9)$

$\mathrm{C}(7)-\mathrm{C}(10)$

$\mathrm{C}(13)-\mathrm{C}(14)$

$\mathrm{C}(13)-\mathrm{O}(5)$

$\mathrm{N}(1)-\mathrm{O}(1)$

$\mathrm{N}(1)-\mathrm{O}(2)$

$\mathrm{N}(2)-\mathrm{O}(3)$

$\mathrm{N}(2)-\mathrm{O}(4)$

Molecule B

$\mathrm{C}(1)-\mathrm{C}(2)$

$\mathrm{C}(1)-\mathrm{C}(6)$

$\mathrm{C}(1)-\mathrm{C}(7)$

$\mathrm{C}(2)-\mathrm{C}(3)$

$\mathrm{C}(2)-\mathrm{N}(2)$

C(3)-C(4)

C(3)-C(12)

C(4)-C(5)

C(4)-C(13)

$\mathrm{C}(5)-\mathrm{C}(6)$

$C(5)-C(11)$

C(6)-N(1)

$\mathrm{C}(7)-\mathrm{C}(8)$

$\mathrm{C}(7)-\mathrm{C}(9)$

$\mathrm{C}(7)-\mathrm{C}(10)$

C(13)-C(14)

$\mathrm{C}(13)-\mathrm{O}(5)$

$\mathrm{N}(1)-\mathrm{O}(1)$

$\begin{array}{lc}\text { Atoms } & \text { Distance }(\AA) \\ \mathrm{C}(6)-\mathrm{N}(13) & 1.476(4) \\ \mathrm{C}(7)-\mathrm{C}(8) & 1.530(5) \\ \mathrm{C}(7)-\mathrm{C}(9) & 1.543(5) \\ \mathrm{C}(7)-\mathrm{C}(10) & 1.536(4) \\ \mathrm{N}(13)-1 \mathrm{O}(13) & 1.228(3) \\ \mathrm{N}(13)-2 \mathrm{O}(13) & 1.220(4) \\ \mathrm{N}(14)-\mathrm{OO}(14) & 1.218(4) \\ \mathrm{N}(14)-2 \mathrm{O}(14) & 1.220(4) \\ \mathrm{N}(15)-\mathrm{O}(15) & 1.200(4) \\ \mathrm{N}(15)-2 \mathrm{O}(15) & 1.202(4)\end{array}$

C(5)-C(11)

$\mathrm{C}(6)-\mathrm{N}(14)$

$\mathrm{C}(7)-\mathrm{C}(8)$

C(7)-C(9)

$\mathrm{C}(7)-\mathrm{C}(10)$

$\mathrm{N}(14)-1 \mathrm{O}(14)$

$\mathrm{N}(14)-2 \mathrm{O}(14)$

$\mathrm{N}(15)-1 \mathrm{O}(15)$

$\mathrm{N}(15)-2 \mathrm{O}(15)$

$1.510(4)$

$1.477(2)$

$1.553(4)$

$1.546(4)$

$1.524(4)$

$1.218(3)$

$1.224(3)$

$1.213(3)$

$1.222(3)$
1.388(3)

$$
\begin{aligned}
& 1.417(18) \\
& 1.407(20) \\
& 1.536(20) \\
& 1.384(19) \\
& 1.478(18) \\
& 1.391(19) \\
& 1.503(21) \\
& 1.395(20) \\
& 1.515(20) \\
& 1.373(20) \\
& 1.522(21) \\
& 1.490(20) \\
& 1.542(26) \\
& 1.546(25) \\
& 1.543(26) \\
& 1.482(24) \\
& 1.198(19) \\
& 1.215(18) \\
& 1.208(19) \\
& 1.211(18) \\
& 1.222(18)
\end{aligned}
$$

\section{$1.396(19)$}

$1.386(20)$

$1.554(20)$

$1.382(20)$

$1.484(19)$

$1.390(20)$

$1.521(23)$

$1.401(22)$

$1.512(21)$

$1.388(21)$

$1.519(23)$

$1.488(21)$

$1.526(27)$

$1.557(25)$

$1.524(29)$

$1.488(27)$

$1.198(20)$

$1.193(21)$

C(5)-C(11)

$\mathrm{C}(6)-\mathrm{N}(1)$

$\mathrm{C}(7)-\mathrm{C}(8)$

Table 11 (continued)

\begin{tabular}{lccc} 
Atoms & Distance $(\AA)$ & Atoms & Distance $(\AA)$ \\
$\mathrm{C}(7)-\mathrm{C}(9)$ & $1.523(24)$ & $\mathrm{N}(1)-\mathrm{O}(1)$ & $1.225(17)$ \\
$\mathrm{C}(7)-\mathrm{C}(10)$ & $1.538(26)$ & $\mathrm{N}(1)-\mathrm{O}(2)$ & $1.226(17)$ \\
$\mathrm{C}(13)-\mathrm{C}(14)$ & $1.497(25)$ & $\mathrm{N}(2)-\mathrm{O}(3)$ & $1.233(18)$ \\
$\mathrm{C}(13)-\mathrm{O}(5)$ & $1.199(18)$ & $\mathrm{N}(2)-\mathrm{O}(4)$ & $1.199(19)$ \\
\hline
\end{tabular}

Table 12. Selected bond angles involving non-hydrogen atoms in compounds (6) $-(8)$ with their e.s.d.s in parentheses

Compound (6)

Atoms

$\mathrm{C}(2)-\mathrm{C}(1)-\mathrm{C}(6)$

$\mathrm{C}(2)-\mathrm{C}(1)-\mathrm{C}(7)$

$\mathrm{C}(6)-\mathrm{C}(1)-\mathrm{C}(7)$

$\mathrm{C}(1)-\mathrm{C}(2)-\mathrm{C}(3)$

$\mathrm{C}(1)-\mathrm{C}(2)-\mathrm{N}(14)$

$\mathrm{C}(3)-\mathrm{C}(2)-\mathrm{N}(14)$

C(2)-C(3)-C(4)

$\mathrm{C}(2)-\mathrm{C}(3)-\mathrm{C}(12)$

C(4)-C(3)-C(12)

$\mathrm{C}(3)-\mathrm{C}(4)-\mathrm{C}(5)$

C(3)-C(4)-N(15)

$\mathrm{C}(5)-\mathrm{C}(4)-\mathrm{N}(15)$

$\mathrm{C}(4)-\mathrm{C}(5)-\mathrm{C}(6)$

$\mathrm{C}(4)-\mathrm{C}(5)-\mathrm{C}(11)$

$\mathrm{C}(6)-\mathrm{C}(5)-\mathrm{C}(11)$

$\mathrm{C}(1)-\mathrm{C}(6)-\mathrm{C}(5)$

$\mathrm{C}(1)-\mathrm{C}(6)-\mathrm{N}(13)$

Angle $(\AA)$
$111.9(2)$
$124.2(2)$
$123.8(2)$
$126.9(2)$
$121.0(2)$
$112.0(2)$
$114.6(2)$
$123.1(3)$
$122.3(3)$
$124.9(2)$
$117.2(2)$
$117.9(2)$
$115.4(2)$
$122.6(3)$
$122.0(3)$
$126.3(2)$
$121.1(2)$

Compound (7)

$\mathrm{C}(2)-\mathrm{C}(1)-\mathrm{C}(6)$

$\mathrm{C}(2)-\mathrm{C}(1)-\mathrm{C}(7)$

$\mathrm{C}(6)-\mathrm{C}(1)-\mathrm{C}(7)$

$\mathrm{C}(1)-\mathrm{C}(2)-\mathrm{C}(3)$

$\mathrm{C}(1)-\mathrm{C}(2)-\mathrm{N}(15)$

$\mathrm{C}(3)-\mathrm{C}(2)-\mathrm{N}(15)$

$\mathrm{C}(2)-\mathrm{C}(3)-\mathrm{C}(4)$

$\mathrm{C}(2)-\mathrm{C}(3)-\mathrm{C}(13)$

$\mathrm{C}(4)-\mathrm{C}(3)-\mathrm{C}(13)$

$\mathrm{C}(3)-\mathrm{C}(4)-\mathrm{C}(5)$

$\mathrm{C}(3)-\mathrm{C}(4)-\mathrm{C}(12)$

$\mathrm{C}(5)-\mathrm{C}(4)-\mathrm{C}(12)$

$\mathrm{C}(4)-\mathrm{C}(5)-\mathrm{C}(5)$

C(4)-C(5)-C(11)

$C(6)-C(5)-C(11)$

Compound (8)

Molecule A

$\mathrm{C}(2)-\mathrm{C}(1)-\mathrm{C}(6)$

$\mathrm{C}(2)-\mathrm{C}(1)-\mathrm{C}(7)$

$\mathrm{C}(6)-\mathrm{C}(1)-\mathrm{C}(7)$

$\mathrm{C}(1)-\mathrm{C}(2)-\mathrm{C}(3)$

$\mathrm{C}(1)-\mathrm{C}(2)-\mathrm{N}(2)$

$\mathrm{C}(3)-\mathrm{C}(2)-\mathrm{N}(2)$

$\mathrm{C}(2)-\mathrm{C}(3)-\mathrm{C}(4)$

$\mathrm{C}(2)-\mathrm{C}(3)-\mathrm{C}(12)$

$\mathrm{C}(4)-\mathrm{C}(3)-\mathrm{C}(12)$

C(3)-C(4)-C(5)

$\mathrm{C}(3)-\mathrm{C}(4)-\mathrm{C}(13)$

$\mathrm{C}(5)-\mathrm{C}(4)-\mathrm{C}(13)$

C(4)-C(5)-C(6)

$\mathrm{C}(4)-\mathrm{C}(5)-\mathrm{C}(11)$

C(6)-C(5)-C(11)

$\mathrm{C}(1)-\mathrm{C}(6)-\mathrm{C}(5)$

$\mathrm{C}(1)-\mathrm{C}(6)-\mathrm{N}(1)$

$\mathrm{C}(5)-\mathrm{C}(6)-\mathrm{N}(1)$

$\mathrm{C}(1)-\mathrm{C}(7)-\mathrm{C}(8)$

$\mathrm{C}(8)-\mathrm{C}(7)-\mathrm{C}(9)$
$110.5(2)$

$125.0(2)$

$124.4(2)$

$126.5(2)$

$120.5(2)$

$113.0(2)$

$118.4(2)$

$121.3(2)$

$120.3(2)$

$119.3(2)$

$120.6(2)$

$120.0(2)$

$118.3(2)$

$120.5(2)$

121.1(2)
$126.0(12)$

$112.4(13)$

$107.4(13)$
Atoms

$\mathrm{C}(5)-\mathrm{C}(6)-\mathrm{N}(13)$

$\mathrm{C}(1)-\mathrm{C}(7)-\mathrm{C}(8)$

$\mathrm{C}(1)-\mathrm{C}(7)-\mathrm{C}(9)$

$\mathrm{C}(1)-\mathrm{C}(7)-\mathrm{C}(10)$

$\mathrm{C}(8)-\mathrm{C}(7)-\mathrm{C}(9)$

$\mathrm{C}(8)-\mathrm{C}(7)-\mathrm{C}(10)$

$\mathrm{C}(9)-\mathrm{C}(7)-\mathrm{C}(10)$

$\mathrm{C}(6)-\mathrm{N}(13)-1 \mathrm{O}(13)$

$\mathrm{C}(6)-\mathrm{N}(13)-2 \mathrm{O}(13) \quad 118.3(3)$

$1 \mathrm{O}(13)-\mathrm{N}(13)-2 \mathrm{O}(13) \quad 124.2(3)$

$\mathrm{C}(2)-\mathrm{N}(14)-1 \mathrm{O}(14) \quad 117.1(3)$

$\mathrm{C}(2)-\mathrm{N}(14)-2 \mathrm{O}(14) \quad 117.5(3)$

$10(14)-\mathrm{N}(14)-2 \mathrm{O}(14) \quad 125.3(3)$

C(4)-N(15)-1O(15) 117.7(3)

$\mathrm{C}(4)-\mathrm{N}(15)-2 \mathrm{O}(15) \quad 119.1(3)$

$1 \mathrm{O}(15)-\mathrm{N}(15)-2 \mathrm{O}(15) \quad 123.2(3)$

$\begin{array}{ll}\mathrm{C}(1)-\mathrm{C}(6)-\mathrm{C}(5) & 126.9(2) \\ \mathrm{C}(1)-\mathrm{C}(6)-\mathrm{N}(14) & 120.4(2) \\ \mathrm{C}(5)-\mathrm{C}(6)-\mathrm{N}(14) & 112.7(2) \\ \mathrm{C}(1)-\mathrm{C}(7)-\mathrm{C}(8) & 112.6(2) \\ \mathrm{C}(1)-\mathrm{C}(7)-\mathrm{C}(9) & 113.3(2) \\ \mathrm{C}(1)-\mathrm{C}(7)-\mathrm{C}(10) & 108.8(2) \\ \mathrm{C}(8)-\mathrm{C}(7)-\mathrm{C}(9) & 103.8(2) \\ \mathrm{C}(8)-\mathrm{C}(7)-\mathrm{C}(10) & 109.8(2) \\ \mathrm{C}(9)-\mathrm{C}(7)-\mathrm{C}(10) & 108.4(2) \\ \mathrm{C}(6)-\mathrm{N}(14)-1 \mathrm{O}(14) & 117.2(2) \\ \mathrm{C}(6)-\mathrm{N}(14)-2 \mathrm{O}(14) & 118.2(2) \\ 1 \mathrm{O}(14)-\mathrm{N}(14)-2 \mathrm{O}(14) & 124.4(2) \\ \mathrm{C}(2)-\mathrm{N}(15)-1 \mathrm{O}(15) & 118.1(2) \\ \mathrm{C}(2)-\mathrm{N}(15)-2 \mathrm{O}(15) & 116.8(2) \\ 1 \mathrm{O}(15)-\mathrm{N}(15)-2 \mathrm{O}(15) & 125.0(2)\end{array}$

109.7(12)

124.3(12)

127.3(12)

$120.0(12)$

$112.6(11)$

$117.0(12)$

$123.9(12)$

$119.0(12)$

$121.0(13)$

$119.4(12)$

$119.5(12)$

$117.3(13)$

$120.9(13)$

$121.8(13)$

$127.7(13)$

$119.8(13)$
$\mathrm{C}(1)-\mathrm{C}(7)-\mathrm{C}(9)$

$\mathrm{C}(1)-\mathrm{C}(7)-\mathrm{C}(10)$

$113.4(13)$

$112.9(13)$

1.480
$C(8)-C(7)-C(10)$ $\mathrm{C}(9)-\mathrm{C}(7)-\mathrm{C}(10)$

$\mathrm{C}(4)-\mathrm{C}(13)-\mathrm{C}(14)$

$\mathrm{C}(4)-\mathrm{C}(13)-\mathrm{O}(5)$

$\mathrm{C}(14)-\mathrm{C}(13)-\mathrm{O}(5)$

$\mathrm{C}(6)-\mathrm{N}(1)-\mathrm{O}(1)$

$\mathrm{C}(6)-\mathrm{N}(1)-\mathrm{O}(2)$

$\mathrm{O}(1)-\mathrm{N}(1)-\mathrm{O}(2)$

$\mathrm{C}(2)-\mathrm{N}(2)-\mathrm{O}(3)$

$\mathrm{C}(2)-\mathrm{N}(2)-\mathrm{O}(4)$

$\mathrm{O}(3)-\mathrm{N}(2)-\mathrm{O}(4)$

Molecule B

$\mathrm{C}(2)-\mathrm{C}(1)-\mathrm{C}(6)$

$C(2)-C(1)-C(7)$

$\mathrm{C}(6)-\mathrm{C}(1)-\mathrm{C}(7)$

$\mathrm{C}(1)-\mathrm{C}(2)-\mathrm{C}(3)$

$C(1)-C(2)-N(2)$

$\mathrm{C}(3)-\mathrm{C}(2)-\mathrm{N}(2)$

$\mathrm{C}(2)-\mathrm{C}(3)-\mathrm{C}(4)$

$\mathrm{C}(2)-\mathrm{C}(3)-\mathrm{C}(12)$

$C(4)-C(3)-C(12)$
$107.4(14)$

104.3(14)

$118.0(13)$

$119.2(14)$

$122.7(15)$

$117.3(13)$

$117.8(14)$

$124.8(15)$

$116.7(13)$

$117.5(13)$

125.6(14)

$111.7(12)$

$124.4(12)$

$123.9(12)$

127.0(13)

$120.7(12)$

$112.2(12)$ 
Table 12. (continued)

Compound (8)

\begin{tabular}{|c|c|c|c|}
\hline Atoms & Angle $(\AA)$ & Atoms & Angle ( $\AA$ \\
\hline$C(3)-C(4)-C(5)$ & $119.6(13)$ & $C(8)-C(7)-C(9)$ & $109.6(2$ \\
\hline$C(3)-C(4)-C(13)$ & $119.8(13)$ & $C(8)-C(7)-C(10)$ & $104.3(19)$ \\
\hline$C(5)-C(4)-C(13)$ & $120.6(13)$ & $C(9)-C(7)-C(10)$ & $103.5(22)$ \\
\hline$C(4)-C(5)-C(6)$ & $118.4(13)$ & $C(4)-C(13)-C(14)$ & $117.5(14)$ \\
\hline$C(4)-C(5)-C(11)$ & $119.0(14)$ & $C(4)-C(13)-O(5)$ & $119.9(15)$ \\
\hline$C(6)-C(5)-C(11)$ & $122.6(14)$ & $C(14)-C(13)-O(5)$ & $122.6(16)$ \\
\hline$C(1)-C(6)-C(5)$ & $125.8(14)$ & $C(6)-N(1)-O(1)$ & $117.0(14)$ \\
\hline$C(1)-C(6)-N(1)$ & $122.6(13)$ & $\mathrm{C}(6)-\mathrm{N}(1)-\mathrm{O}(2)$ & $117.7(14)$ \\
\hline$C(5)-C(6)-N(1)$ & $111.6(13)$ & $\mathrm{O}(1)-\mathrm{N}(1)-\mathrm{O}(2)$ & $125.2(16)$ \\
\hline$C(1)-C(7)-C(8)$ & $108.4(13)$ & $C(2)-N(2)-O(3)$ & $119.2(13)$ \\
\hline$C(1)-C(7)-C(9)$ & $112.3(13)$ & $\mathrm{C}(2)-\mathrm{N}(2)-\mathrm{O}(4)$ & $115.3(13)$ \\
\hline$C(1)-C(7)-C(10)$ & $113.8(14)$ & $\mathrm{O}(3)-\mathrm{N}(2)-\mathrm{O}(4)$ & $125.4(15)$ \\
\hline$C(8)-C(7)-C(9)$ & $108.0(14)$ & & \\
\hline$C(8)-C(7)-C(10)$ & $110.0(15)$ & Molecule D & \\
\hline$C(9)-C(7)-C(10)$ & $104.2(15)$ & $C(2)-C(1)-C(6)$ & $111.9(12)$ \\
\hline$C(4)-C(13)-C(14)$ & $117.9(14)$ & $C(2)-C(1)-C(7)$ & $123.5(12)$ \\
\hline$C(4)-C(13)-O(5)$ & $120.0(15)$ & $C(6)-C(1)-C(7)$ & $124.5(12)$ \\
\hline$C(14)-C(13)-O(5)$ & $122.0(16)$ & $C(1)-C(2)-C(3)$ & $125.8(13)$ \\
\hline$C(6)-N(1)-O(1)$ & $117.0(15)$ & $C(1)-C(2)-N(2)$ & $122.2(12)$ \\
\hline$C(6)-N(1)-O(2)$ & $116.1(15)$ & $C(3)-C(2)-N(2)$ & $111.9(12)$ \\
\hline$O(1)-N(1)-O(2)$ & $126.8(17)$ & $C(2)-C(3)-C(4)$ & $117.9(13)$ \\
\hline$C(2)-N(2)-O(3)$ & $118.8(13)$ & $C(2)-C(3)-C(12)$ & $121.3(13)$ \\
\hline$C(2)-N(2)-O(4)$ & $117.1(13)$ & $C(4)-C(3)-C(12)$ & $120.7(13)$ \\
\hline $\mathrm{O}(3)-\mathrm{N}(2)-\mathrm{O}(4)$ & $124.1(15)$ & $C(3)-C(4)-C(6)$ & $120.8(12)$ \\
\hline & & $C(3)-C(4)-C(13)$ & $119.5(12)$ \\
\hline Molecule C & & $C(5)-C(4)-C(13)$ & $119.8(12)$ \\
\hline$C(2)-C(1)-C(6)$ & $111.2(12)$ & $C(4)-C(5)-C(6)$ & $117.4(11)$ \\
\hline$C(2)-C(1)-C(7)$ & $125.3(12)$ & $C(4)-C(5)-C(11)$ & $119.5(12)$ \\
\hline$C(6)-C(1)-C(7)$ & $123.5(12)$ & $C(6)-C(5)-C(11)$ & $123.0(12)$ \\
\hline$C(1)-C(2)-C(3)$ & $126.2(12)$ & $C(1)-C(6)-C(5)$ & $126.1(12)$ \\
\hline $\mathrm{C}(1)-\mathrm{C}(2)-\mathrm{N}(2)$ & $121.7(12)$ & $C(1)-C(6)-N(1)$ & $120.8(11)$ \\
\hline$C(3)-C(2)-N(2)$ & $112.1(11)$ & $C(5)-C(6)-N(1)$ & $112.8(11)$ \\
\hline$C(2)-C(3)-C(4)$ & $118.0(12)$ & $C(1)-C(7)-C(8)$ & $112.2(12)$ \\
\hline$C(2)-C(3)-C(12)$ & $122.0(13)$ & $C(1)-C(7)-C(9)$ & $108.2(12)$ \\
\hline$C(4)-C(3)-C(12)$ & $120.0(13)$ & $C(1)-C(7)-C(10)$ & $113.2(13)$ \\
\hline$C(3)-C(4)-C(5)$ & $121.3(13)$ & $C(8)-C(7)-C(9)$ & $108.3(13)$ \\
\hline$C(3)-C(4)-C(13)$ & $119.9(13)$ & $C(8)-C(7)-C(10)$ & $103.8(14)$ \\
\hline$C(5)-C(4)-C(13)$ & $118.6(13)$ & $C(9)-C(7)-C(10)$ & $111.1(14)$ \\
\hline$C(4)-C(5)-C(6)$ & $116.7(13)$ & $C(4)-C(13)-C(14)$ & $117.6(13)$ \\
\hline$C(4)-C(5)-C(11)$ & $122.0(14)$ & $C(4)-C(13)-O(5)$ & $119.5(14)$ \\
\hline$C(6)-C(5)-C(11)$ & $121.3(14)$ & $C(14)-C(13)-O(5)$ & $122.9(15)$ \\
\hline$C(1)-C(6)-C(5)$ & $126.4(13)$ & $C(6)-N(1)-O(1)$ & $117.4(12)$ \\
\hline$C(1)-C(6)-N(1)$ & $121.4(12)$ & $C(6)-N(1)-O(2)$ & $117.5(12)$ \\
\hline$C(5)-C(6)-N(1)$ & $112.1(12)$ & $O(1)-N(1)-O(2)$ & $125.0(13)$ \\
\hline$C(1)-C(7)-C(8)$ & $112.6(15)$ & $\mathrm{C}(2)-\mathrm{N}(2)-\mathrm{O}(3)$ & $115.0(13)$ \\
\hline$C(1)-C(7)-C(9)$ & $110.4(19)$ & $\mathrm{C}(2)-\mathrm{N}(2)-\mathrm{O}(4)$ & $118.3(13)$ \\
\hline$C(1)-C(7)-C(10)$ & $115.9(17)$ & $\mathrm{O}(3)-\mathrm{N}(2)-\mathrm{O}(4)$ & $126.6(15)$ \\
\hline
\end{tabular}

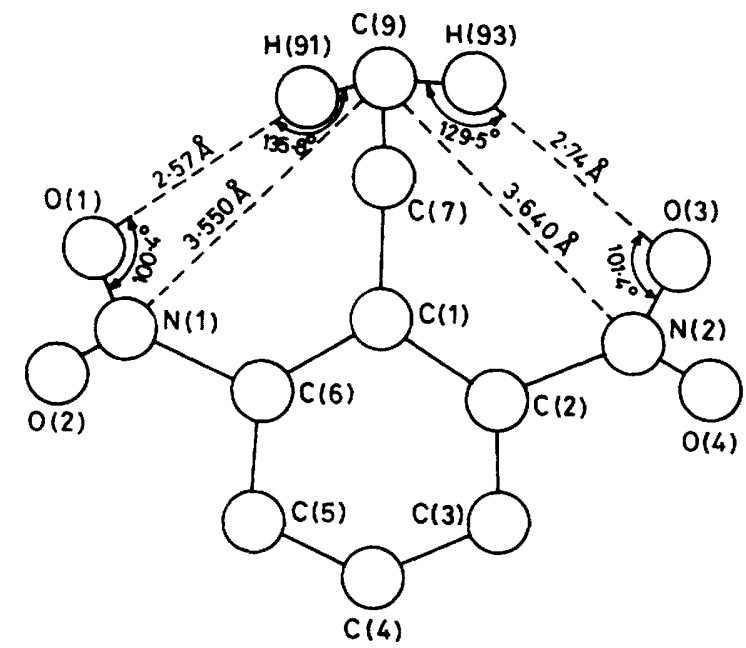

Figure 3. Actual geometry of hydrogen abstraction for compound (6)

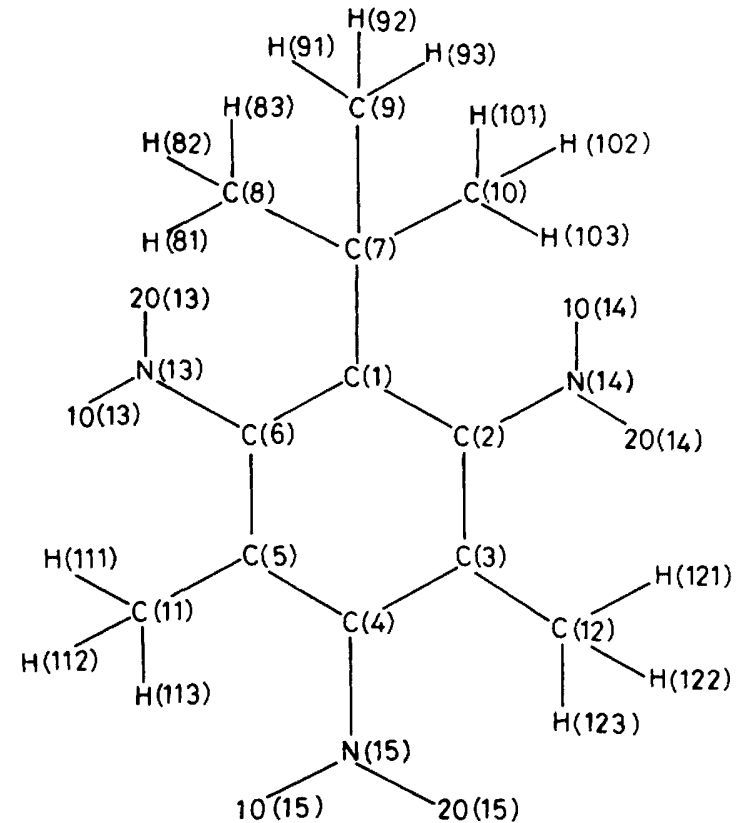

Figure 4. Numbering scheme for compound (6)

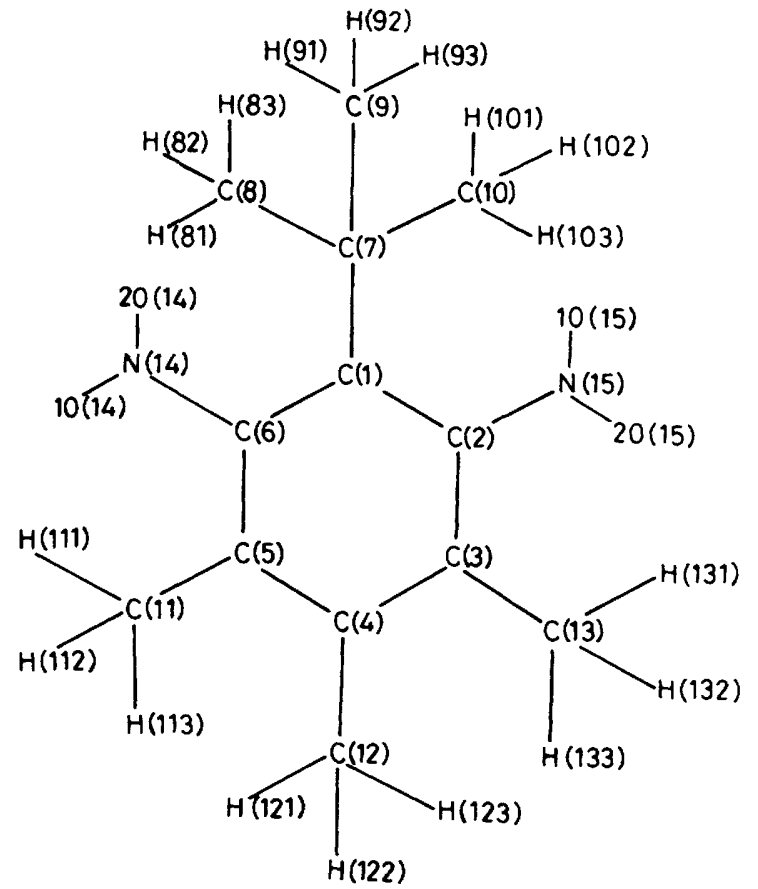

Figure 5. Numbering scheme for compound (7)

In order for the $\mathrm{C}$ and $\mathrm{N}$ radicals to combine after initial hydrogen abstraction, the t-butyl group has to undergo a rotation about the $\mathrm{C}(1)-\mathrm{C}(7)$ bond either in the positive or in the negative direction. It is relevant to mention that there is nonrigid librational motion about the vectors $\mathrm{C}(1)-\mathrm{C}(7)$, $\mathrm{C}(2)-\mathrm{N}(2)$, and $\mathrm{C}(6)-\mathrm{N}(1)$ with amplitudes $6.2(5), 6.7(11)$, and 6.8(11) degrees, respectively. The thermal motion analysis program THMB developed by Trueblood ${ }^{8}$ was used for this analysis. Rotation in the positive direction would bring $C(9)$ into the proximity of $\mathrm{N}(1)$ and rotation in the negative direction 


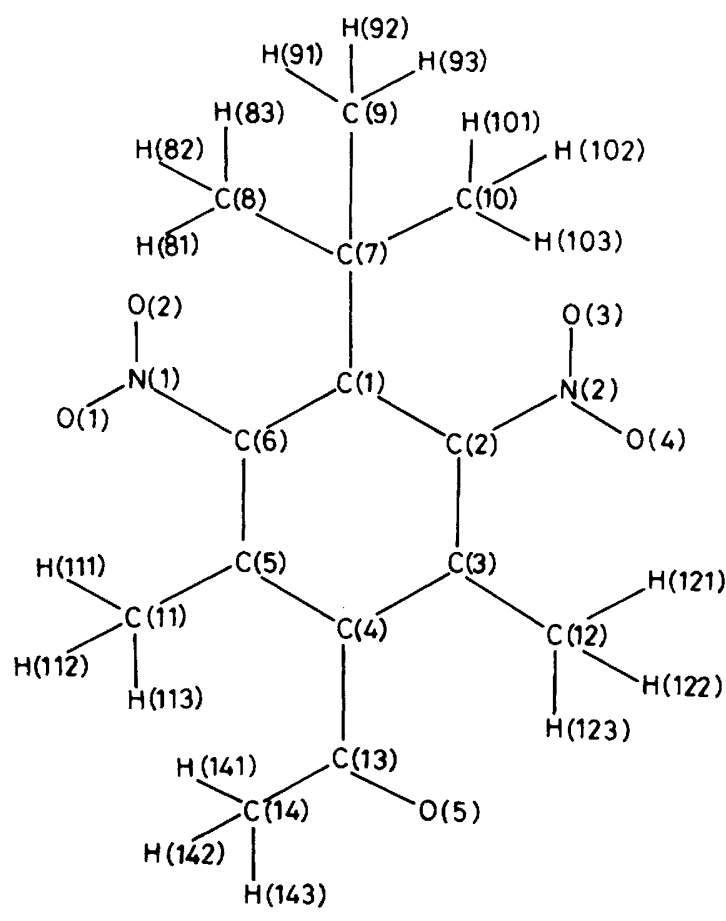

Figure 6. Common numbering scheme for molecules A, B, C, and D of (8)
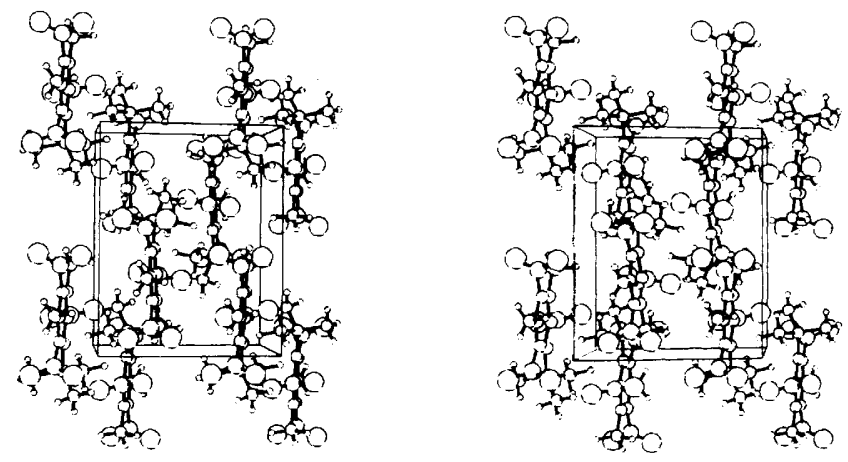

Figure 7. Stereoscopic drawing of the packing of the molecules of compound (6) viewed along the z-axis. The axial directions are $a \rightarrow$, $b \uparrow$, and $c$ down out of the plane of the paper

would bring $C(9)$ nearer to $N(2)$. The hydrogen atoms that would be abstracted in these two cases are different and they are $H(91)$ by $O(1)$ and $H(93)$ by $O(3)$, respectively. Now the question is whether such a rotation will be tolerated by the environment in the crystal lattice and if so in which direction the rotation about $\mathrm{C}(1)-\mathrm{C}(7)$ is preferred. Here considerations of the intermolecular interactions would be relevant. Intermolecular short contacts were calculated for the rotation of the t-butyl group in both directions at $5^{\circ}$ intervals and this was calculated up to $\pm 30^{\circ}$, as this much rotation is sufficient to bring the $\mathrm{C}$ and $\mathrm{N}$ radicals close enough to interact. Intermolecular van der Waals energies were not computed for these rotations. Instead the sum $S=\Sigma\left(d_{0}-d_{c}\right)^{2}$ was calculated for each of these rotations (Table 7), where $d_{0}$ is the standard value of van der Waal's sum for different atom pairs, and $d_{c}$ is the corresponding intermolecular short contact on rotation of the t-butyl group. This criterion was employed by Williams ${ }^{9}$ for the solution of crystal structures with known molecular structures and lattice dimensions. It is reasonable to expect that the smaller $\mathbf{S}$ corresponds to the energetically more favourable situation. Thus the greater value of $S$ for the rotation by -30 suggests that the rotation in the positive direction is more favourable in the solid state and this brings $C(9)$ into the proximity of $\mathrm{N}(1)$ for radical combination. It therefore seems reasonable to conclude that in crystals of $(6)$, the most probable reaction is abstraction of $\mathrm{H}(91)$ by $\mathrm{O}(1)$ followed by combination of $\mathrm{C}(9)$ and $\mathrm{N}(1)$ radicals. Figure 3 shows the actual geometry for hydrogen abstraction in compound (6). It may be emphasized that the intramolecular $\mathrm{C}-\mathrm{H} \ldots \mathrm{O}$ and $\mathrm{N}-\mathrm{O} \cdot . \mathrm{H}$ angles between the hydrogens of the benzylic methyl groups and the nitro groups show that the nitro groups cannot interact with the benzylic methyl groups in the solid state, the values obtained for the appropriate angles being much less favourable than those involving the t-butyl group protons and adjacent nitro groups. The conclusions derived from crystallographic data are in agreement with the experimental observations. ${ }^{4}$

Following a similar approach, it is concluded that, for compound (7), abstraction of $H(91)$ by $O(1)$ followed by bond formation between $\mathrm{C}(9)$ and $\mathrm{N}(1)$ leads to the product. The nonrigid librational motion about the vectors $\mathrm{C}(1)-\mathrm{C}(7), \mathrm{C}(2)-\mathrm{N}(2)$, and $\mathrm{C}(6)-\mathrm{N}(1)$ in compound (7) is $7.0(15), 8.8(9)$, and $8.0(10)$ degrees, respectively. The $\operatorname{sum} \Sigma\left(d_{o}-d_{c}\right)^{2}$ for rotation in both directions is given in Table 7.

Compound (8) contains four independent molecules in the asymmetric unit, and the relative geometries of the reacting groups (the t-butyl and the two ortho-nitro groups) for molecules A, B, and D are the same as that of compound (6) and (7). Consequently, all generalizations made for (6) and (7) are applicable for molecules A, B, and D of (8). Regarding molecule $C$ of $(8)$, the orientation of the t-butyl group with respect to the phenyl ring is different from that of the other three molecules of the same compound and also from that in (6) and (7) as indicated by the torsional angles (Table 8). As seen in Table 6 , there are only five $\mathrm{O} \cdots \mathrm{H}$ contacts less than $3 \AA$ for molecule $\mathrm{C}$ of $(8)$. When we consider the relative orientation of the $\mathrm{C}-\mathrm{H}$ and $\mathrm{N}-\mathrm{O}$ groups (Table 6) they are quite favourable for two sets of atoms: (i) $\mathrm{O}(1)$ with $H(81)$ and (ii) $O(2)$ with $H(93)$, two nearly symmetrical possibilities. But when we take into consideration the distance $(\mathrm{O} \cdots \mathrm{H}$ and $\mathrm{C} \cdots \mathrm{N})$ as well as directional criteria $(\mathrm{C}-\mathrm{H} \cdots \mathrm{O}$ and $\mathrm{N}-\mathrm{O} \cdots \mathrm{H})$ it is most probably the hydrogen $\mathrm{H}(103)$ which would be abstracted by $\mathrm{O}(3)$, followed by the combination of $\mathrm{C}(10)$ and $\mathrm{N}(2)$ (Table 6).

Regarding compound (8), it may be mentioned that the accuracy of the structural parameters is much less than that of compounds (6) and (7). Furthermore, it has been found that the non-rigid librational motion about the $\mathrm{C}(1)-\mathrm{C}(7)$ vector in molecule $\mathrm{C}$ of compound (8) is much larger $\left[25.9(9)^{\circ}\right]$ than in other molecules in the asymmetric unit which tend to have a value of $c a$. $6.8^{\circ}$. Thus, it seems difficult to arrive at definite conclusions about the hydrogen being abstracted in molecule $C$ of compound (8).

The results presented in this paper show that there can be some specificity in the hydrogen-abstraction reactions of nitro compounds (6)-(8). It is obvious that one cannot experimentally demonstrate the specificity implied from our analyses. However, we hope that the considerations in this study are general and will be useful in analysing more demanding situations.

\section{Experimental}

The details of irradiation of the above compounds and isolation of the products have been reported earlier. ${ }^{4}$ 

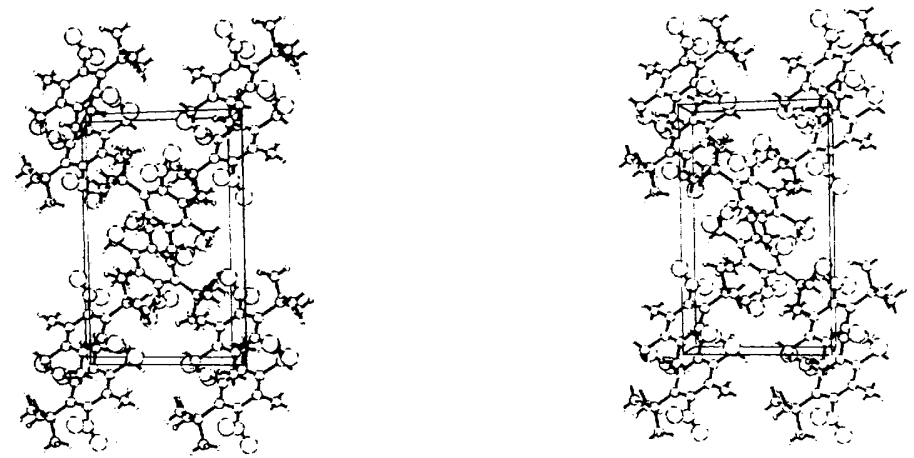

Figure 8. Stereoscopic drawing of the packing of the molecules of compound (7) viewed along the z-axis. The axial directions are as for compound (6)
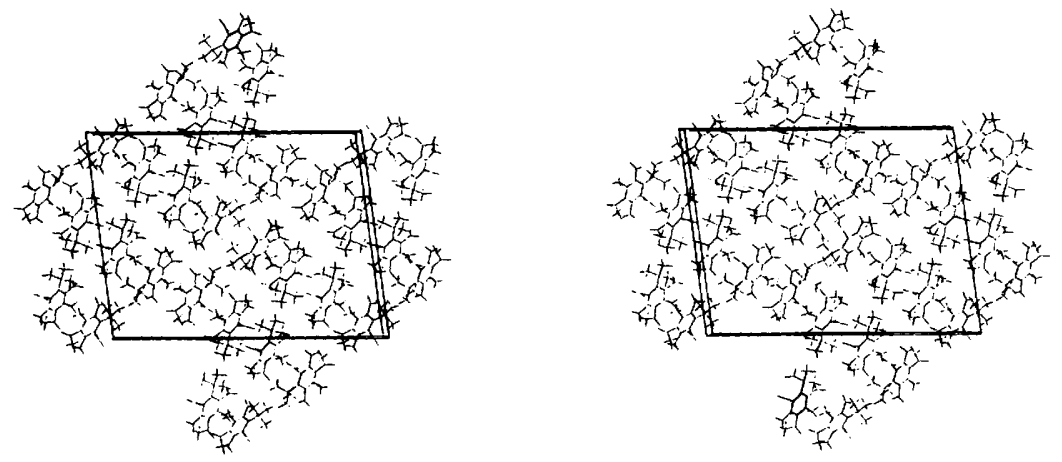

Figure 9. Stereoscopic drawing of the packing of the molecules of compound (8) viewed along the $y$-axis. The axial directions are $a \uparrow, b$ down out of the plane of the paper, and $c \rightarrow$

X-Ray Crystallographic Analysis of Compounds (6)-(8).Colourless crystals were obtained by slow evaporation of an ethyl acetate solution of (6) (1-t-butyl-3,5-dimethyl-2,4,6trinitrobenzene, 'musk xylene'), (lit., ${ }^{4}$ m.p. $112-113^{\circ} \mathrm{C}$ ), and of a benzene solution of both (7) (1-t-butyl-3,4,5-trimethyl-2,6dinitrobenzene, 'musk tibetene'), (lit., ${ }^{4}$ m.p. $\left.138^{\circ} \mathrm{C}\right)$, and (8) (1-tbutyl-4-acetyl-3,5-dimethyl-2,6-dinitrobenzene, 'musk ketone'), (lit., ${ }^{4}$ m.p. $137^{\circ} \mathrm{C}$ ). The cell constants and possible space groups were obtained from oscillation and Weissenberg photographs. Accurate cell dimensions and intensity data were obtained from an Enraf-Nonius CAD-4 diffractometer using either monochromated $\mathrm{Cu}-K_{\alpha}$ radiation [for (7)] or monochromated Mo$K_{\alpha}$ radiation [for $(6)$ and $(8)$ ] in $\omega / 2 \theta$ scan mode. Three standard reflections were measured for every sixty reflections and no significant changes in the intensities of these reflections were observed.

All the structures were solved by the direct methods program MULTAN-80. ${ }^{10}$ Isotropic least-squares refinement and further refinement with anisotropic thermal parameters for (6) and (7) was carried out using SHELX-76. ${ }^{11}$ Hydrogen atoms were initially fixed using difference Fourier synthesis and refined for positional and isotropic thermal parameters.

Compound (8) contains four independent molecules in the asymmetric unit. The first E-map gave 78 non-hydrogens out of the $\mathbf{8 4}$ for the four molecules. The remaining $\mathbf{6}$ atoms were fixed at stereochemically reasonable positions. All the 72 hydrogens of the four molecules were also fixed stereochemically. Refinement of the positional and anisotropic thermal parameters of non-hydrogens and isotropic thermal parameters for hydrogens was carried out by the block diagonal leastsquares program SFLS ${ }^{12}$ written by Shiono and modified by B. S. Reddy. Hamilton's ${ }^{13}$ significance test was applied to assess whether hydrogen atoms could be refined. The refinement of hydrogens was significant even at the $0.5 \%$ level. The details of the refinement are given in Table 9. It may be mentioned that the accuracy of the structure determination of compound (8) is much less than that of compounds (6) and (7). Final positional parameters of the non-hydrogen atoms are listed in Table 10, bond lengths in Table 11, and bond angles in Table 12 . Numbering schemes relevant to Tables 10, 11 , and 12 are shown in Figures 4-6. The crystal structures are solely stabilized by van der Waals forces, having no intermolecular hydrogen bonds. The packing arrangements of the molecules of compounds $(6)-(8)$ are illustrated in Figures $7-9$, respectively. Illustrations were made by PLUTO. ${ }^{14}$

Tables of anisotropic thermal parameters, parameters of hydrogen atoms, and selected bond lengths and angles are available as a Supplementary Publication [SUP No. 56512 (23 pp.)]*

\section{Acknowledgements}

One of us (K. P.) thanks the Department of Atomic Energy, Government of India, for a Junior Research Fellowship. We thank Dr. M. N. Ponnuswamy for intensity data collections and Prof. J. Trotter for his interst in the work.

\footnotetext{
* For details of the Supplementary Publications Scheme, see J. Chem. Soc., Perkin Trans. 2, 1986, issue 1. Structure factor tables are available
} on request from the editorial office.

\section{References}

I D. Döpp, Chem. Ber., 1971, 104, 1035

2 D. Döpp. Chem. Ber., 1971, 104, 1043.

3 D. Döpp and E. Brugger, Liehigs Ann. Chem.. 1979. 554. 
4 D. Döpp and K. H. Sailer, Tetrahedron Lett., 1975, 1129; D. Döpp and K. H. Sailer, Chem. Ber., 1975, 108, 3483.

5 D. Döpp, Tetrahedron Lett., 1971, 2757, and unpublished work.

6 H. A. Morrison, 'The Chemistry of the Nitro and Nitroso Groups,' ed. H. Feuer, Part 1, Interscience, New York, 1969, pp. 165.

7 W. K. Appel, Z. Q. Jiang, J. R. Scheffer, and L. Walsh, J. Am. Chem. Soc., 1983, 105, 5354; J. Trotter, Acta Crystallogr., Sect. B., 1983, 39, 373; J. R. Scheffer and A. A. Dzakpasu, J. Am. Chem. Soc., 1978, 100 , 2163.

$8 \mathrm{~K}$. N. Trueblood, personal communication, 1982.

9 D. E. Williams, Acta Crystallogr., Sect. A., 1969, 25, 464.

10 P. Main, S. J. Fiske, S. E. Hull, L. Lessinger, G. Germain, J.-P. Declercq, and M. M. Woolfson, MULTAN-80. A System of Computer Programs for the Automatic Solution of Crystal
Structures from X-ray Diffraction Data, Univs. of York, England, and Louvain, Belgium, 1980.

11 G. M. Sheldrick, SHELX-76. Program for Crystal Structure Determination, Univ. of Cambridge, England, 1976.

12 R. Shiono, personal communication, 1968.

13 W. C. Hamilton, Acta Crystallogr., 1965, 18, 502; W. C. Hamilton, 'International Tables for $X$-ray Crystallography,' vol. 4, p. 286 , Kynoch Press, Birmingham, 1974.

14 W. D. S. Motherwell and W. Clegg, Program for the production of crystal and molecular illustrations, Crystallographic Data Centre, Cambridge, England, 1978.

Received 8th July 1985; Paper 5/1145 